\title{
Mind and Machine. The New Spaces of Robots and Digitization
}

\author{
Bruce Janz \\ (University of Central Florida, Orlando; bruce.janz@ucf.edu) \\ ORCID: 0000-0003-3122-1618
}

\section{André Schmiljun}

(Humboldt-University of Berlin - Adam Mickiewicz University of Poznań; andre_schmiljun@icloud.com) ORCID: 0000-0002-9415-8495

This special volume of Ethics in Progress addresses the issue of mind and machines. Machines have always been a tool or technical instrument for human beings to facilitate and to accelerate processes through mechanical power. The same applies to robots nowadays - the next step in the evolution of machines. They already clean our houses, mow our lawns, hunt terrorists via drones and transport heavy loads. Robots are somehow "enchanting to humans", as Maciej Musiał (2018) puts it. Over the course of the last few years, robot usage in society has expanded enormously, and they now carry out a remarkable number of tasks for us. In many sectors, like finance, medicine, education or agriculture, transportation and factory production, it is likely that there are at least a handful of jobs for human workers that will sooner or later be replaced by robots or autonomous solutions.

Furthermore, robot scientists like Kevin Warwick believe that they are able to develop an artificial brain, comparable to our human brain, that is able to generate a robotic mind. According to Nick Bostrom, we are far "from having reached the physical limits of technology" (Bostrom 2014, 110). If that is indeed the case, we are on the eve of a historic revolution that will change everything we know right now. But not only robots have an impact on our life. It is digitization in its entirety, including smart applications and games, that confronts us with new spaces. And, the realization that robots are embodied cognitive systems present us with even more new spaces for impact, along with new anxieties for how to live in a world in which we cannot entirely deduce the behaviour of complex systems from their initial programming. We have been accustomed to think of robots as extensions of ourselves, either in the benign sense of being tools or the threatening sense of being our moral shadow. The new spaces in which robots exist, though, may not be as either the fulfilment of our desires or the manifestation of our fears, but as something entirely different - another emergent cognition, holding forth the possibility that what 
it means to be human, and what it means to be a robot, depends on ahuman conditions, that is, the conditions of existence defined not by internal programming or ideas about fundamental identity, but by the autopoiesis of complex interaction between these emergent systems. Our questions about life with robots and other complex technologies, then, may be less about finding the algorithm or programming formula to guarantee a desired behavioural result in our creations, and more about finding the adaptive strategies to learn from interactions with entities which will inevitably become more unpredictable to us as time goes on.

This volume tries to broaden our understanding of a philosophical field - robots and digitization - that is still in its infancy in terms of it research and literature. It is divided into three sections:

In the first section, Janina L. Samuel, Katarzyna Ginszt, Volker Thiel, Artur Jocz and Peter Remmers concentrate on the interaction of robots and human beings in society, asking for example about the psychological perspective on social robots, anthropomorphism and the ethical implications of Artificial Intelligence (AI).

In the second section, Catrin Misselhorn, Paweł Łupkowski \& Katarzyna Wasielewska, and André Schmiljun consider the theoretical foundations for artificial morality and the need of morality in human-robot interaction.

In the third section, Patrícia da Silva Leite \& Deborah Andrade Torquato Schimidt illustrate that digital games are social objects based on our culture and society, shaping our world perspective. Finally, the Joanna Pankau's article focuses on the socio-spatial accessibility of the city, considering the phenomenon of the digitally expanding urban environment: new-media mobility conditions, intermedia dataflow, hybrid space. Such mobile applications can be sensible solutions, especially for people with disabilities. Małgorzata Bogaczyk-Vormayr's paper on plantality studies with the focus on microphotography closes the third section and reorients a reader's attention towards the link between life structures and microscopy, which reflects the non-naturalistic, antiillustrative, and interpretative character of artistic microphotography.

Last but not least, the varia section includes a paper authored by Weiyu Yang which reports on recent studies on traditional ethics in China and Japan.

We thank our many contributors and reviewers. Without their support, this volume would not have been possible. A special thanks goes as well to Ewa Nowak, Marcin Jan Byczyński and Stephen Dersley, who not only edited all the texts over countless hours, but also provided useful recommendations and ideas for this volume. 


\section{References}

Bostrom N. 2014. Superintelligence. Paths, Dangers, Strategies. Oxford: University Press. Musiał M. 2018. Enchanting Robots. Intimacy, Magic, and Technology. Switzerland:

Palgrave Macmillan - Springer. 


$$
\begin{gathered}
\text { Bruce Janz (Orlando) } \\
\text { André Schmiljun (Berlin - Poznań) }
\end{gathered}
$$

\section{Mind and Machine: The New Spaces of Robots and Digitization}

Abstract: Machines have always been a tool or technical instrument for human beings to facilitate and to accelerate processes through mechanical power. The same applies to robots nowadays - the next step in the evolution of machines. Over the course of the last few years, robot usage in society has expanded enormously, and they now carry out a remarkable number of tasks for us. It seems we are on the eve of a historic revolution that will change everything we know right now. But not only robots have an impact on our life. It is digitization in its entirety, including smart applications and games, that confronts us with new spaces. This special volume of Ethics in Progress tries to broaden our understanding of a philosophical field - robots and digitization - that is still in its infancy in terms of it research and literature.

Keywords: robots; robot ethics; machine ethics; moral competence; robot morality; artificial morality; moral implementation; care robots; social robots; anthropomorphism; uncanny valley; digitization; digital games; smart applications; people with disabilities; microphotography; Adam Snerg-Wiśniewski; Georg Lind; Matthias Scheutz; Isaac Asimov; Wendell Wallach; Colin Allen; Bertram F. Malle.

Ethics in Progress (ISSN 2084-9257). Vol. 10 (2019). No. 2, Art. \#1, pp. 4-7. Creative Commons BY-SA 4.0

DOI:10.14746/eip.2019.2.1

1 The article was copy-edited by a native speaker, Stephen Dersley, with the translation supported by the grant 261/WCN/2019/1 "Wsparcie dla Czasopism Naukowych" (2019-2020) in order to promote original Polish research worldwide. 